\title{
Alta frecuencia de pancreatitis aguda asociada a patología biliar en niños chilenos
}

\author{
JUAN CARLOS PATTILLO S. ${ }^{1}$, GUSTAVO MONTECINOS A. ${ }^{1}$, \\ MARÍA JOSÉ LUQUE H. ${ }^{2}$, PAUL HARRIS D. ${ }^{2}$ \\ 1. Sección Cirugía Pediátrica, División de Cirugía, Facultad de Medicina, Pontificia Universidad Católica de Chile. \\ 2. Unidad de Gastroenterología, Hepatología y Nutrición, División de Pediatría, Facultad de Medicina, Pontificia Universidad \\ Católica de Chile.
}

\begin{abstract}
Elevated frequency of Acute Pancreatitis Associated to biliary Illness in Chilean Children

Acute Pancreatitis (AP) in children presents significant morbimortality. Most common etiologies in this age group are trauma, systemic illness and idiopathic pancreatitis. This is different from adult AP, where lithiasis and alcohol consumption are the predominant causes. In Chile, where billiary disease is highly prevalent, there is little information regarding AP among children. Objective: To determine the main clinical characteristics of acute pancreatitis in a group of chilean children. Patients and Methods: A retrospective study (1998-2008) of patients hospitalized with the diagnosis of AP. The diagnosis was confirmed by elevation of pancreatic enzymes and through images. Demographic data, etiology, complications and resolutions, need for parenteral nutrition, and use of antibiotics were examined. Results: Eighteen patients were identified (8,3 \pm 4 y.o.). Etiology of AP was listed as: lithiasis and alterations of biliary duct: 38,8\%, idiopathic: $22,2 \%$, secondary to medications: 22,2\% and other: $16.8 \%$. Two patients presented peripancreatic infected collections; a similar number formed pancreatic pseudocysts. Six patients (33,3\%) required one type of surgical procedure as part of their treatment. Two-thirds of all patients required treatment in ICU. One half of the patients required parenteral nutrition, and two thirds received IV antibiotics. The median length of hospital stay was 20 days (ave 24,9 $\pm 14,3$ ds). There was no mortality in this serie. Conclusions: Unlike previously described, biliary AP was the most common cause in this serie. Biliary pathology should be actively studied among chilean children with AP.

(Key words: Acute pancreatitis, cholelithiasis, children).

(Rev Chil Pediatr 2011; 82 (6): 525-530
\end{abstract}

\section{RESUMEN}

La pancreatitis aguda (PA) en niños presenta una morbimortalidad considerable. Las etiologías más frecuentes en este grupo etario son la PA secundaria a trauma, por enfermedades sistémicas y la PA idiopática, a diferencia de la población adulta en que predomina la litiasis biliar y el consumo de alcohol. En Chile, donde la patología biliar es altamente prevalente, existe escasa información clínica respecto de la PA en niños. Objetivo: Determinar las características clínicas de la pancreatitis aguda en un grupo de niños chilenos. Método: Estudio

Trabajo recibido el 06 de julio de 2011, devuelto para corregir el 23 de agosto de 2011, segunda versión el 21 de septiembre de 2011, aceptado para publicación el 15 de octubre de 2011.

Correspondencia a:

Dr. Juan Carlos Pattillo S.

E-mail: jpattill@med.puc.cl 
retrospectivo (1998-2008) de pacientes hospitalizados con PA. Se confirmó el diagnóstico por elevación de enzimas pancreáticas e imágenes; se obtuvieron los datos demográficos y se analizó: etiología, complicaciones y su resolución, necesidad de nutrición parenteral y uso de antibióticos. Resultados: Se identificaron 18 pacientes (8,3 \pm 4 años). Etiología de la PA: litiasis y alteraciones anatómicas de la vía biliar (38,8\%), idiopática (22,2\%), drogas (22,2\%), otras (16.8\%). Dos pacientes presentaron colecciones peripancreáticas infectadas; igual número evolucionó con formación de pseudoquistes pancreáticos. Seis pacientes (33,3\%) requirieron algún tipo de procedimiento quirúrgico como parte de su tratamiento. Dos tercios de los pacientes ingresaron a la Unidad de Pacientes Críticos. La mitad de los pacientes recibió nutrición parenteral y dos tercios antibióticos endovenosos. La mediana de hospitalización fue de 20 días (promedio 24,9 \pm 14,3 días). No hubo mortalidad en la serie. Conclusiones: A diferencia de lo descrito en la literatura, la PA biliar fue la etiología más frecuente en esta serie. La patología biliar debe ser estudiada activamente en niños chilenos con PA.

(Palabras clave: Pancreatitis aguda, colelitiasis, niños).

Rev Chil Pediatr 2011; 82 (6): 525-530

\section{Introducción}

La pancreatitis aguda (PA) en niños, aunque poco frecuente, es una patología que requiere de manejo intrahospitalario, multidisciplinario, y que se asocia a cifras considerables de mortalidad, que en series antiguas alcanzaba el $21 \%{ }^{1}$. Actualmente, la mortalidad descrita es de alrededor de un $10 \%{ }^{2,3}$. Artículos recientes señalan que la incidencia de la PA en niños ha aumentado en los últimos años ${ }^{2,4}$. Las causas más frecuentes de PA en pediatría son el trauma, las enfermedades sistémicas, el uso de drogas, la PA idiopática y la patología biliar, incluyendo alteraciones anatómicas de la vía biliar y litiasis ${ }^{2,5,6}$. Existe consenso en que la PA idiopática ocupa un lugar relevante en la lista de causas (en ocasiones, primer lugar), postulándose mutaciones en genes involucrados en la secreción pancreática como una explicación plausible ${ }^{7}$. Otras causas menos frecuentes de PA en niños incluyen las infecciones, principalmente virales (paperas, hepatitis A, rotavirus, hepatitis E, varicela, adenovirus y coxsackie) y ocasionalmente bacterianas (Mycoplasma pneumoniae y Moraxella catarrhalis), las alteraciones del metabolismo (hipertrigliceridemia e hipercalcemia) y algunas condiciones genéticas hereditarias en las que existe pérdida de los mecanismos de protección de la acción de las enzimas pancreáticas ${ }^{8}$. La mayoría de las series pediátricas proviene de países desarrollados; estos estudios no reportan la patología biliar (litiasis o malforma- ciones de la vía biliar) como una causa relevante de PA, a diferencia de lo que ocurre en la población adulta, en la que la litiasis biliar, junto con el consumo de alcohol, son las principales etiologías de $\mathrm{PA}^{9}$.

Existen escasos datos respecto de las características de los pacientes pediátricos con PA en nuestro país, donde la patología biliar es altamente prevalente. El objetivo de este artículo es describir las características clínicas y demográficas de un grupo de niños chilenos con PA y determinar si la presencia de patología biliar es una causa relevante de PA.

\section{Pacientes y Método}

\section{Pacientes}

Estudio retrospectivo. Se analizó el registro de egresos del Servicio de Pediatría del Hospital Clínico de la Pontificia Universidad Católica de Chile durante el período 1998-2008, incluyéndose dentro del estudio a todos los pacientes con el diagnóstico de Pancreatitis Aguda.

\section{Revisión}

Se revisaron las fichas clínicas y electrónicas de los pacientes. La certificación del diagnóstico se realizó mediante una elevación de amilasa o lipasa de al menos 2 veces el valor normal, o alteraciones compatibles con PA en una tomografía abdominal (TAC) de abdomen. En los pacientes que presentaron episodios re- 
currentes de PA, sólo se consideró el primer evento para efectos de este estudio.

\section{Análisis}

Dentro de las variables tabuladas se incluyeron: presentación clínica, etiología, días de hospitalización, necesidad de admisión a Unidades de Paciente Crítico (UPC), imágenes utilizadas, uso de antibióticos y uso de nutrición parenteral, gravedad según score de Balthazar (tabla 1$)^{10}$, procedimientos quirúrgicos y complicaciones, entre otras. Se completó el seguimiento con controles ambulatorios realizados post alta. Se realizó un análisis descriptivo de las variables estudiadas, en cuanto a frecuencia y porcentajes.

\section{Resultados}

\section{Pacientes}

Se identificaron 18 pacientes que cumplieron criterios de inclusión. De ellos 10 fueron niños (55,6\%) y 8 niñas $(44,4 \%)$. La edad promedio de presentación fue de 8,3 \pm 4 años (rango 1-15 años). Cuatro pacientes presentaban comorbilidad previo al episodio de PA: epilepsia y leucemia linfoblástica aguda (2 pacientes cada una).

\section{Cuadro clínico}

Todos los pacientes estudiados presentaron dolor abdominal al ingreso, el resto de los síntomas se detalla en la tabla 2. El total de los pacientes de la serie contaba con niveles de amilasa plasmática, cuyo valor promedio fue de $1532 \mathrm{U} / \mathrm{L}$, rango 226-5 $320 \mathrm{U} / \mathrm{L}$ (valor normal 10-220 U/L), el 95\% de los pacientes presentó elevación de este marcador enzimático al momento del diagnóstico. En un 78\% de los casos se disponía de medición de lipasa plasmática, siendo su valor promedio 1168 U/L, rango 82-4 305 U/L (valor normal menor a $60 \mathrm{U} / \mathrm{L})$.

\section{Imágenes}

En relación al estudio imagenológico durante la hospitalización, a un 77,8\% de los pacientes se les realizó ecotomografía abdominal, a un $88,9 \%$ tomografía computada de abdomen y pelvis; y a un 27,8\% resonancia nu- clear magnética de la vía biliar. Respecto de la clasificación de la PA por imágenes, un 31,3\% de los pacientes se presentó con un Balthazar E. Los pacientes clasificados como Balthazar C y D siguieron en frecuencia con un $25 \%$ de los casos cada uno de los grupos.

\section{Etiología}

La distribución de causas está descrita en la tabla 3. Dentro de la categoría biliar, que fue la más frecuente, se incluyeron cinco pacientes $(27,7 \%)$ con patología litiásica, uno de

Tabla 1. Clasificación de gravedad de PA por TAC

\begin{tabular}{|c|c|}
\hline Clasificación & Hallazgo TAC \\
\hline A & Páncreas normal \\
\hline B & Edema pancreático \\
\hline C & $\begin{array}{l}\text { Inflamación del páncreas y/o de la grasa } \\
\text { peripancreática }\end{array}$ \\
\hline D & Colección líquida peripancreática única \\
\hline E & $\begin{array}{l}\text { Dos o más colecciones peripancreáticas y/o } \\
\text { aire retroperitoneal }\end{array}$ \\
\hline
\end{tabular}

PA: pancreatitis aguda. TAC: tomografía axial computarizada.

Tabla 2. Presentación clínica de 18 niños con PA

\begin{tabular}{|lcc|}
\hline Clínica & $\mathbf{n}$ & $\mathbf{( \% )}$ \\
\hline Dolor abdominal & 18 & $(100)$ \\
\hline Vómitos & 15 & $(83,3)$ \\
\hline Diarrea & 4 & $(22,2)$ \\
\hline Ictericia & 1 & $(5,6)$ \\
Hematoquezia & 1 & $(5,6)$ \\
\hline
\end{tabular}

PA: pancreatitis aguda.

Tabla 3. Etiología de PA en 18 niños

\begin{tabular}{|lcc|}
\hline Etiología & $\mathbf{n}$ & $\mathbf{( \% )}$ \\
Biliar & 7 & $(38,8)$ \\
$\quad$ - litiasis & 5 & $(27,7)$ \\
- alteración anatómica & 2 & $(11,1)$ \\
Idiopática & 4 & $(22,2)$ \\
\hline Drogas & 4 & $(22,2)$ \\
Litiasis Wirsung & 1 & $(5,6)$ \\
Hematoma duodenal & 1 & $(5,6)$ \\
Trauma & 1 & $(5,6)$ \\
\hline Total & 18 & $(100)$ \\
\hline
\end{tabular}

PA: pancreatitis aguda. 
ellos desarrolló PA luego de colecistectomía electiva, y 2 pacientes $(11,1 \%)$ con quiste del colédoco, ambos con diagnóstico histológico confirmado luego de su resolución quirúrgica. De los cuatro niños con PA secundaria a drogas, el 50\% fue secundario a ácido valproico, y la otra mitad fue atribuida al uso de L-asparaginasa como droga de quimioterapia. El único paciente de la serie con PA atribuida a trauma abdominal presentó un hematoma en la cola del páncreas, resuelto con laparotomía exploradora y drenaje de hematoma.

\section{Evolución y tratamientos}

Doce pacientes $(66,7 \%)$ recibieron antibióticos durante su estadía, con un promedio de tratamiento de 13,7 días. En 10 pacientes (55,6\%) se indicó nutrición parenteral. Ello debido a que no fue posible utilizar la vía enteral por la gravedad clínica, la distención abdominal o a la presencia de íleo. Dos tercios de los pacientes ingresaron a la UPC durante de su hospitalización. Seis niños (33,3\%) fueron sometidos a uno más procedimientos invasivos y/o cirugía, ya sea para el manejo etiológico o de las complicaciones de la PA. Dentro de los procedimientos realizados se incluyeron: CPRE, drenaje colecciones infectadas y pseudoquiste pancreático, y drenaje de hematoma pancreático. Dos pacientes presentaron colecciones peripancreáticas infectadas; uno de ellos fue drenado por vía laparoscópica, el otro paciente pudo ser manejado con una punción bajo TAC. Igualmente, dos niños presentaron pseudoquistes pancreáticos, uno de ellos requirió drenaje por vía endoscópica, mientras que el segundo paciente fue manejado en forma médica. Dos pacientes fueron sometidos a una resección de quiste de colédoco y hépatoentero anastomosis en Y de Roux. Todos los pacientes fueron evaluados durante su hospitalización por médicos de las especialidades de Pediatría, Gastroenterología, Nutrición y Cirugía Pediátrica, además de los médicos de la Unidad de Pacientes Críticos, cuando fue necesario. La mediana de hospitalización fue de 20 días (promedio 24,9 \pm 14,3 días; rango 5-53 días). No hubo mortalidad en la serie durante la hospitalización ni en el seguimiento vía ficha clínica electrónica.

\section{Discusión}

Este estudio, con las limitaciones de un estudio retrospectivo, presenta algunos aspectos diferentes respecto de las pocas series reportadas en niños con PA provenientes de países desarrollados. En nuestra serie, la patología de la vía biliar es causa de la mayoría de los episodios de PA (casi 40\%). Dentro de este grupo, existe una preponderancia de la patología litiásica, la que podría estar explicada por las características demográficas de la población chilena ${ }^{11}$. Esto difiere de lo publicado en series pediátricas en la literatura internacional. También es notoria la alta incidencia de quistes del colédoco, llegando a un $11,1 \%$ del total de pacientes, lo que podría explicarse en el contexto de un sesgo de derivación de patología de nivel terciario a nuestra institución. En series modernas se reporta una incidencia de patología biliar de 9-30\% ${ }^{4-6,12}$. Ziegler sin embargo, señala a la patología biliar litiásica (litiasis pigmentada) como la principal etiología de PA en niños, pero en su serie encontramos una alta incidencia de pacientes con enfermedades hematológicas, como anemia de células falciformes ${ }^{13}$, lo que no es extrapolable a la realidad de nuestro país. Llama la atención la baja incidencia de PA secundaria a trauma en nuestra serie en comparación a otros reportes, esto podría estar explicada por las características de derivación de nuestro centro hospitalario dónde el trauma es poco habitual. La incidencia encontrada para PA idiopática es semejante a la reportada en la literatura, alrededor de un $20 \%$, sin embargo, la incidencia de PA secundaria a drogas $(22,2 \%)$ es muy superior a los datos disponibles en la literatura, que reportan valores entre $3,2 \%$ y $13 \%{ }^{2,14}$ del total de pacientes. Algunos autores postulan que el ácido valproíco es la droga que más frecuentemente se asocia a PA en niños ${ }^{14,15}$, lo cual está en concordancia con los hallazgos de nuestro estudio.

La presencia de dolor abdominal y vómitos son elementos muy frecuentes en la clínica de la PA, alcanzando el $82 \%$ y $53 \%$, respectivamente, en grupos reportados previamente ${ }^{16}$. En nuestra serie, todos los pacientes ingresaron con dolor abdominal al Servicio de Urgencia. Aunque se describe clásicamente el íleo adiná- 
mico como parte del cuadro clínico de la PA, un 22\% de nuestros pacientes presentó diarrea al ingreso.

El tratamiento de la PA es esencialmente médico, ya sea en población pediátrica o adulta. Aproximadamente un $80 \%$ de las PA en niños son manejados sólo con medidas de soporte $^{17}$. Los procedimientos invasivos y la cirugía están fundamentalmente indicados para el manejo de las complicaciones y, en algunos casos, de la etiología cuando ésta es aguda. Los pacientes analizados en el período descrito presentaron una tasa de resolución exclusivamente médica en el 66,6\%; un tercio de ellos requirieron procedimientos invasivos durante su hospitalización. Cinco de nuestros pacientes requirieron cirugía $(27,8 \%)$, cifra inferior en comparación con otros autores que reportan resolución quirúrgica en el 38-59\% de los ca$\operatorname{sos}^{12,18}$. Uno de nuestros pacientes que requirió cirugía fue resuelto completamente por vía mínimamente invasiva: primero aseo quirúrgico de colecciones infectadas, luego cistogastrostomía endoscópica para el drenaje de pseudoquiste pancreático y finalmente, colecistectomía laparoscópica con colangiografía intraoperatoria para el manejo de colelitiasis. Este paciente fue incluido en una serie de pacientes adultos sometidos a necrosectomía y drenaje de abscesos por vía laparoscópica publicado en la literatura nacional ${ }^{16}$. Está ampliamente documentado en la literatura que la resolución de las complicaciones de la PA con técnicas de cirugía mínimamente invasiva es segura y eficiente, esto es válido tanto en adultos como en población pediátrica ${ }^{18-24}$.

La mayoría de nuestros pacientes recibió antibióticos de amplio espectro y requirió manejo en UPC. Esto último, considerando además la necesidad de cirugía en un porcentaje significativo de pacientes, es consistente con un curso grave, en pacientes complejos. Existe consenso en que la PA en pediatría debe ser manejada por profesionales de diferentes áreas en un equipo interdisciplinario ${ }^{17}$. Cobra especial relevancia el soporte nutricional de estos pacientes: más de la mitad de nuestros pacientes necesitó nutrición parenteral. En general favorecemos el uso de la vía enteral para el soporte nutricional de nuestros pacientes y consideramos la nutrición parenteral en casos de hiepertensión intraabdominal, íleo o shock séptico. La pancreatitis aguda en niños mantiene cifras considerables de morbilidad.

A diferencia de lo descrito en la literatura, la PA biliar fue la etiología más frecuente en esta serie. La patología biliar debe ser estudiada activamente en niños chilenos con PA.

\section{Referencias}

1.- Weizman Z, Durie P: Acute pancreatitis in childhood. J Pediatr 1988; 113 (1): 24-9.

2.- Nydegger A, Heine RG, Ranuh R, Gegati-Levy $R$, Crameri J, Oliver MR: Changing incidence of acute pancreatitis: 10-year experience at the Royal Children's Hospital, Melbourne. J Gastroenterol Hepatol 2007; 22 (8): 1313-6

3.- Benifla M, Weizman Z: Acute pancreatitis in childhood: analysis of literature data. J Clin Gastroenterol 2003; 37 (2): $100-2$.

4.- Werlin SL, Kugathasan S, Frautschy BC: Pancreatitis in children. J Pediatr Gastroenterol Nutr 2003; 37: 591-5.

5.- Kandula L, Lowe ME: Etiology and outcome of acute pancreatitis in infants and toddlers. J Pediatr 2008; 152 (1): 106-10, 110.e1. Epub 2007 Oct 24.

6.- Bai HX, Lowe ME, Husain SZ: What have we learned about acute pancreatitis in children? JPGN 2011; 52: 262-70.

7.- Nydegger A, Couper RT, Oliver MR: Childhood pancreatitis. J Gastroenterol Hepatol 2006; 21 (3): 499-509.

8.- Whitcomb DC: Genetic aspects of pancreatitis. Annu Rev Med 2010; 61:413-24.

9.- Martínez J, Zúñiga A, Guzmán S, et al: Acute severe pancreatitis. Analysis of mortality and morbidity. Rev Med Chile 1991; 119 (6): 659-69.

10.- Emil J: Balthazar, Acute Pancreatitis: Assessment of Severity with Clinical and CT Evaluation. Radiology 2002; 223: 603-13.

11.- Miquel JF, Covarrubias C, Villaroel L, et al: Genetic epidemiology of cholesterol cholelithiasis among Chilean Hispanics, Amerindians, and Maoris. Gastroenterol 1998; 115 (4): 937-46.

12.- DeBanto JR, Goday PS, Pedroso MR, et al: Acute pancreatitis in children. Am J Gastroenterol 2002; 97: 1726-31.

13.- Ziegler DW, Long JA, Philippart AI, Klein MD: Pancreatitis in childhood. Experience with 49 patients. Ann Surg 1988; 207 (3): 257-61.

14.- Houben ML, Wilting I, Stroink H, van Dijken PJ: 
PATTILLO J. y cols.

Pancreatitis, complicated by a pancreatic pseudocyst associated with the useof valproic acid. Eur J Paediatr Neurol 2005; 9: 77-80.

15.- Vaca C, et al: Pancreatitis aguda grave y pseudoquiste pancreático por uso de drogas en niños: Presentación de tres casos clínicos y revisión de la literatura. Rev Chil Pediatr 2001; 72 (3): 235-43.

16.- Stringer D: Pancreatitis and pancreatic trauma. Semin Pediatr Surg 2005; 14 (4): 239-46.

17.- Yachha SK, Chetri K, Saraswat VA, et al: Management of childhood pancreatic disorders: a multidisciplinary approach. J Pediatr Gastroenterol Nutr 2003; 36 (2): 206-12.

18.- Synn AY, Mulvihill SJ, Fonkalsrud EW: Surgical management of pancreatitis in childhood. J Pediatr Surg 1987; 22: 628-32.

19.- Funke R, Donoso A, Rondanelli M, Pattillo JC, et al: Necrosectomía laparoscópica en pancreatitis aguda. Rev
Chil Cir 2010; 62 (5): 470-5.

20.- Saad DF, Gow KW, Cabbabe S, et al: Laparoscopic cystogastrostomy for the treatment of pancreatic pseudocysts in children. J Pediatr Surg 2005; 40 (11): e13-7.

21.- Pamoukian VN, Gagner M: Laparoscopic necrosectomy for acute necrotizing pancreatitis. J Hepatobiliary Pancreat Surg 2001; 8 (3): 221-3.

22.- Mathew A, Biswas A, Meitz KP: Endoscopic necrosectomy as primary treatment for infected peripancreatic fluid collections (with video). Gastrointest Endosc 2008; 68 (4): 776-82.

23.- Bucher P, Pugin F, Morel P: Minimally invasive necrosectomy for infected necrotizing pancreatitis. Pancreas 2008; 36 (2): 113-9.

24.- Seitz G, Warmann S, Kirschner H-J: Laparoscopic cystojejunostomy as a treatment option for pancreatic pseudocysts in children-a case report. J Pediatr Surg 2006; 41: E33-5. 